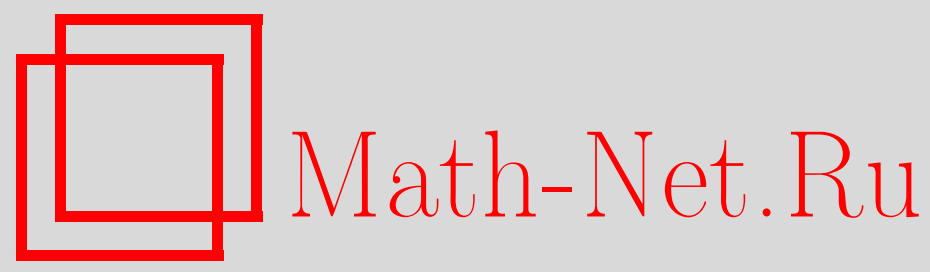

Н. М. Тимофеев, С. Т. Туляганов, Задачи типа аддитивной проблемы делителей, Матем. заметки, 1998, том 64, выпуск 3, 443-456

DOI: https://doi.org/10.4213/mzm1416

Использование Общероссийского математического портала Math-Net.Ru подразумевает, что вы прочитали и согласны с пользовательским соглашением http://www . mathnet.ru/rus/agreement

Параметры загрузки:

IP: 54.92 .164 .108

26 апреля 2023 г., 13:18:08

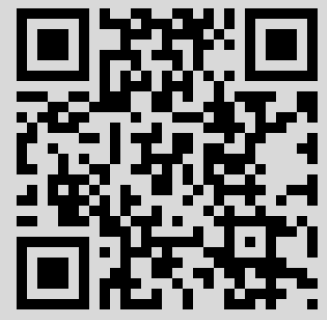


УДК 511.3

\title{
ЗАДАЧИ ТИПА АДДИТИВНОЙ ПРОБЛЕМЫ ДЕЛИТЕЛЕЙ
}

\author{
Н. М. Тимофеев, С. Т. Туляганов
}

Для мультипликативных функций $f(n)$, удовлетворяющих условиям: $f(n) \geqslant 0$, $f\left(p^{r}\right) \leqslant A^{r}, A>0$, для любого $\varepsilon>0$ существуют постоянные $A_{\varepsilon}, \alpha>0$ такие, что $f(n) \leqslant A_{\varepsilon} n^{\varepsilon}$ и $\sum_{p \leqslant x} f(p) \ln p \geqslant \alpha x,-$ доказано соотношение

$$
\sum_{n \leqslant x} f(n) \tau(n-1)=C(f) \sum_{n \leqslant x} f(n) \ln x(1+o(1)) .
$$

Здесь $\tau(n)$ - число делителей $n, C(f)$ - постоянная.

Библиографоия: 13 названий.

Пусть $f(n)$ - мультипликативная функция, т.е. $f(n m)=f(n) f(m)$ для любых взаимно простых $n, m, f(1)=1$. В работе исследуется поведение при $x \rightarrow \infty$ суммы

$$
S(f, x)=\sum_{n \leqslant x} f(n) \tau(n-1),
$$

где $\tau(n)$ - число делителей $n$. Основной целью является доказательство при возможно более слабых ограничениях на $f(n)$ соотношения

$$
S(f, x)=C(f) \sum_{n \leqslant x} f(n) \ln x(1+o(1)) .
$$

Если $f(n)=\tau_{k}(n)$ - число представлений $n$ в виде $k$ сомножителей, то эта задача хорошо известна в теории чисел и носит название аддитивной проблемы делителей. Впервые ее безусловное решение при любом $k$ было получено Ю. В. Линником [1].

В дальнейшем рассматривались различные обобщения этой задачи: А. И. Виноградов [2] исследовал случай, когда $f(n)=\tau_{k}^{m}(n)$, Л.И. Уфимцевой [3] найдена асимптотика $S(f, x)$, если $f(n) \geqslant 0$ и $f(p)=f_{0}$ - целое число, в работе Б.М. Бредихина и Л.И. Уфимцевой [4] изучен случай, когда $f(p)$ "в среднем" равна $f_{0}$. Если $|f(n)| \leqslant 1$, то полное исследование $S(f, x)$ проведено в работе [5]. М. Б. Хрипунова [6] рассмотрела случай $f(n)=\tau_{k}(n)$, где $|f(n)| \leqslant 1$, а $\tau_{k}(n)$ - число представлений $n$ в виде произведения $k$ сомножителей.

Основной результат работы следуюший.

Работапервого автора вьполнена при финансовой поддержке Российского фонда фундаментальных исследований. 
Tеорема. Пусть мультипликативная функиия $f(n) \geqslant 0$ удовлетворяет следуюшим условиям: существует число А такое, что $f\left(p^{r}\right) \leqslant A^{r}, r \geqslant 1 ;$ для любого $\varepsilon>0$ существует константа $A_{\varepsilon}$ такая, что при всех $n f(n) \leqslant A_{\varepsilon} n^{\varepsilon} ;$ существует число $\alpha>0$ такое, что при $x \geqslant x_{0}$

$$
\sum_{p \leqslant x} f(p) \ln p \geqslant \alpha x
$$

Тогда имеет место (1) с постоянной

$$
C(f)=\frac{\zeta(2) \zeta(3)}{\zeta(6)} \sum_{i=1}^{k} \frac{1}{\varphi\left(\delta_{i}\right)} E\left(f, \delta_{i}\right) F\left(f, \delta_{i}\right),
$$

где $(s)$ - дзета-функиия Римана,

$$
\begin{gathered}
E(f, \delta)=\prod_{p \mid \delta} \frac{p(p-1)}{p^{2}-p+1}\left(1+\sum_{r=1}^{\infty} \frac{f\left(p^{r}\right)}{p^{r}}\right)^{-1} \\
F(f, \delta)=\prod_{p \nmid \delta}\left(1+\sum_{r=1}^{\infty} \frac{f\left(p^{r}\right) \chi_{\delta}^{*}\left(p^{r}\right)}{p^{r}} \frac{(p-1)^{2}}{p^{2}-p+1}\right)\left(1+\sum_{r=1}^{\infty} \frac{f\left(p^{r}\right)}{p^{r}}\right)^{-1}
\end{gathered}
$$

$\varphi(n)-$ функиия Эйлера, $\chi_{\delta_{i}}^{*}(i=1, \ldots, k)$ - те примитивнье характеры, для коmopbux

$$
\sum_{p} \frac{f(p)\left(1-\chi_{\delta_{i}}^{*}(p)\right)}{p}<\infty
$$

$1 \leqslant \delta_{1}<\delta_{2} \leqslant \cdots \leqslant \delta_{k}-u x$ модули.

ЗАмЕчАниЕ. Будет показано, что число примитивных характеров $k$, для которых ряд (3) сходится, не превосходит $A / \alpha$.

В качестве следствия можно получить решение некоторых аддитивных задач.

СледствиЕ 1. Пусть $\mathscr{P}$ - некоторое множество степеней простых чисел,

$$
\sum_{\substack{p \leqslant x \\ p \in \mathscr{P}}} \ln p>\alpha x, \quad \alpha>0, \quad x \geqslant x_{0},
$$

$f$ - мультипликативная функиия,

$$
f\left(p^{k}\right)= \begin{cases}1, & \text { если } p^{k} \in \mathscr{P} \\ 0 & \text { в противном случае. }\end{cases}
$$

Тогда справедливо (1) с

$$
C(f)=\frac{\zeta(2) \zeta(3)}{\zeta(6)} \sum_{k=1}^{k} \frac{1}{\varphi\left(\delta_{i}\right)} E_{\delta_{i}}(\mathscr{P}) F_{\delta_{i}}(\mathscr{P}),
$$


$2 \partial e$

$$
\begin{gathered}
E_{\delta}(\mathscr{P})=\prod_{p \nmid \delta} \frac{p(p-1)}{p^{2}-p+1}\left(1+\sum_{\substack{r=1 \\
p^{r} \in \mathscr{P}}}^{\infty} \frac{1}{p^{r}}\right)^{-1}, \\
F_{\delta}(\mathscr{P})=\prod_{p \nmid \delta}\left(1+\sum_{\substack{r=1 \\
p^{r} \in \mathscr{P}}}^{\infty} \frac{\chi_{\delta}^{*}\left(p^{r}\right)}{p^{r}} \frac{(p-1)^{2}}{p^{2}-p+1}\right)\left(1+\sum_{\substack{r=1 \\
p^{r} \in \mathscr{P}}}^{\infty} \frac{1}{p^{r}}\right)^{-1},
\end{gathered}
$$

$\chi_{\delta_{i}}^{*}(i=1, \ldots, k)-$ примитивные характеры, для которых

$$
\sum_{p \in \mathscr{P}} \frac{1-\chi_{\delta_{i}}^{*}(p)}{p}<\infty, \quad k \leqslant \frac{1}{\alpha} .
$$

В следствии 1 найдена асимптотика для числа решений уравнений $n-k s=1, k, s \in \mathbb{N}$, $n \leqslant x$, у которых все степени простых чисел, входящих в каноническое разложение, содержатся в множестве $\mathscr{P}$. В частном случае, когда $\mathscr{P}$ состоит из степеней 2 , всех степеней простых чисел вида $4 k+1$ и четных степеней простых чисел вида $4 k+3$, мы приходим к следующему результату, доказанному в [7].

СлЕДСТВИЕ 2. Имеет место соотношение

$$
\sum_{\substack{n \leqslant x \\ n=m^{2}+k^{2}}} \tau(n-1)=C \sum_{\substack{n \leqslant x \\ n=m^{2}+k^{2}}} 1 \cdot \ln x(1+o(1)),
$$

$2 \partial e$

$$
C=\frac{\zeta(2) \zeta(3)}{\zeta(6)} \prod_{p \equiv 1(\bmod 4)} \frac{p(p-1)}{p^{2}-p+1} \prod_{p \equiv 3(\bmod 4)} \frac{\left(p^{2}+1\right)(p-1)}{p^{2}\left(p^{2}-p+1\right)}
$$

При доказательстве будет часто использоваться следующий результат Шиу [8].

ЛЕмма 1 (Теорема Шиу). Пусть $f(n)$ - мультипликативная функиия, $\left|f\left(p^{r}\right)\right| \leqslant$ $A^{r} ;$ для любого $\varepsilon>0$ существует $A_{\varepsilon}$ такое, что $|f(n)| \leqslant A_{\varepsilon} n^{\varepsilon}$ при $n \geqslant 1$. Тогда для $0<\alpha, \beta<1 / 2,0<a \leqslant k,(a, k)=1, a, k \in \mathbb{N}$

$$
\sum_{\substack{x-y<n \leqslant x \\ n \equiv a(\bmod k)}}|f(n)| \ll \frac{y}{\varphi(k) \ln x} \exp \left(\sum_{\substack{p \leqslant x \\ p \nmid k}} \frac{|f(p)|}{p}\right)
$$

равномерно по $а, k$ и у таким, что $k \leqslant y^{1-\alpha}, x^{\beta}<y \leqslant x$.

Далее будем пользоваться следуюшими обозначениями: $P(x)=\prod_{p \leqslant x} p, d \mid P(x)$ означает, что все простые делители $d$ не превосходят $x$,

$$
\Pi_{f}(x)=\prod_{p \leqslant x}\left(1+\sum_{r=1}^{\infty} \frac{f\left(p^{r}\right)}{p^{r}}\right) .
$$


Лемма 2. Пусть $z_{0} \leqslant z \leqslant \sqrt{x} / \ln ^{\beta} x=Q, d_{1}\left|P(\sqrt{z}), d_{2}\right| P(z),\left(d_{2}, P(\sqrt{z})\right)=1$, $\left(d_{3}, P(z)\right)=1 ; a-$ фиксированное число, $f(n)$ - комплекснозначная мультипликативная функиия, $|f(n)|$ удовлетворяет условиям теоремы. Если $B \geqslant 3+2 A^{2}+3 \gamma$, mo

$$
\Delta(z)=\sum_{\substack{d_{1} d_{2} d_{3} \leqslant Q \\\left(d_{1} d_{2} d_{3}, a\right)=1}} \frac{1}{\varphi\left(d_{3}\right)}\left|\sum_{\substack{n \leqslant x \\ n \equiv a\left(\bmod d_{1} d_{2}\right)}} f(n)-\frac{1}{\varphi\left(d_{2}\right)} \sum_{\substack{n \leqslant x \\\left(n, d_{3}\right)=1}} f(n)\right| \ll R,
$$

где $R=x(\ln z)^{-\gamma} \Pi_{|f|}(x)$ и $\gamma-$ любая положительная постоянная.

ДокАЗАТЕЛЬСТво. Часть суммы $\Delta(z)$, в которой $d_{1} d_{2}>u$, после применения леммы 1 будет

$$
\begin{aligned}
& \ll \frac{x}{\ln x} \sum_{\substack{d_{1} d_{2} d_{3} \leqslant Q \\
d_{1} d_{2}>u}} \frac{1}{\varphi\left(d_{1} d_{2} d_{3}\right)} \Pi_{|f|}(x) \\
& \ll \frac{x}{\ln x} \Pi_{|f|}(x) \frac{1}{\ln u} \sum_{d_{1} d_{2} d_{3} \leqslant Q} \frac{\ln d_{1} d_{2}}{\varphi\left(d_{1} d_{2} d_{3}\right)} \ll \frac{x}{\ln u} \ln z \Pi_{|f|}(x) .
\end{aligned}
$$

Заметим, что в условиях теоремы

$$
\exp \sum_{p \leqslant x} \frac{|f(p)|}{p} \asymp \Pi_{|f|}(x) .
$$

Возьмем $u=\exp \left(\ln ^{\gamma+1} z\right)$. Если $u>Q$, то эта часть суммы равна 0 . Значит, в любом случае оцениваемая часть равна $O(R)$. Представим $n=n_{1} n_{2} n_{3}$, где $n_{1} \mid P\left(y_{1}\right)$, $n_{2} \mid P\left(y_{2}\right),\left(n_{2}, P\left(y_{1}\right)\right)=1,\left(n_{3}, P\left(y_{2}\right)\right)=1, y_{1}<y_{2}$. Покажем, что с точностью до $O(R)$ можно считать, что $u_{1}<n_{1} \leqslant u_{2}, v_{1}<n_{3} \leqslant v_{2}$, где $u_{1}, u_{2}, v_{1}, v_{2}$ - некоторые параметры $\leqslant x^{1 / 3}$, зависящие от $z$ и $x$. Часть оцениваемой суммы с $n_{1} \leqslant u \leqslant x^{1 / 3}$ после применения леммы 1 будет

$$
\begin{aligned}
& \ll \sum_{d_{1} d_{2} d_{3} \leqslant Q} \frac{1}{\varphi\left(d_{1} d_{2} d_{3}\right)} \sum_{n_{1} \leqslant u_{1}} \frac{\left|f\left(n_{1}\right)\right|}{n_{1}} \frac{x}{\ln x} \exp \left(\sum_{y_{1}<p \leqslant x} \frac{|f(p)|}{p}\right) \\
& \ll x \ln ^{A} u_{1} \exp \left(-\sum_{p \leqslant y_{1}} \frac{|f(p)|}{p}\right) \Pi_{|f|}(x) \ll x \Pi_{|f|}(x) \frac{\ln ^{A} u_{1}}{\ln ^{\alpha} y_{1}} .
\end{aligned}
$$

Если $\ln ^{A} u_{1} \leqslant \ln ^{\alpha} y_{1} \cdot \ln ^{-\gamma} z$, то оцениваемая часть равна $O(R)$. Действуя аналогично и учитьвая (2), получим, что часть суммы, в которой $n_{2} \leqslant v_{1} \leqslant x^{1 / 3}$, будет

$$
\ll x\left(\frac{\ln v_{1}}{\ln y_{1}}\right)^{A} \exp \left(-\sum_{y_{1}<p \leqslant y_{2}} \frac{|f(p)|}{p}\right) \Pi_{|f|}(x) \ll \frac{\ln ^{A} v_{1}}{\ln ^{A} y_{1}}\left(\frac{\ln y_{1}}{\ln y_{2}}\right)^{\alpha} \Pi_{|f|}(x) .
$$

Если

$$
\left(\ln v_{1}\right)^{A} \ll \frac{\ln ^{A+\alpha} y_{2}}{\ln ^{\alpha} y_{1}}(\ln z)^{-\gamma}
$$


то и эта часть суммы будет равна $O(R)$. Оценим часть суммы, в которой $n_{1} \geqslant u_{2}$. Разобьем ее на две: в первой $u_{2} \leqslant n_{1}<x^{1 / 3}$, во второй $n_{1} \geqslant x^{1 / 3}$. Для оценки первой снова применим лемму 1. Получим, что она

$$
\begin{aligned}
& \ll \frac{x}{\ln x} \sum_{d_{1} d_{2} d_{3} \leqslant Q} \frac{1}{\varphi\left(d_{1} d_{2} d_{3}\right)} \sum_{u_{2}<n_{1}} \frac{\left|f\left(n_{1}\right)\right|}{n_{1}} \exp \left(\sum_{y_{1}<p \leqslant x} \frac{|f(p)|}{p}\right) \\
& \ll \frac{x}{\ln u_{2}} \sum_{n_{1}} \frac{\left|f\left(n_{1}\right)\right|}{n_{1}} \ln n_{1} \exp \left(\sum_{y_{1}<p \leqslant x} \frac{|f(p)|}{p}\right) \\
& \ll \frac{x}{\ln u_{2}} \sum_{\substack{p \leqslant y_{1} \\
r \geqslant 1}} \frac{\left|f\left(p^{r}\right)\right| \ln p^{r}}{p^{r}} \sum_{n_{1}} \frac{\left|f\left(n_{1}\right)\right|}{n_{1}} \exp \left(\sum_{y_{1}<p \leqslant x} \frac{|f(p)|}{p}\right) \ll \frac{x}{\ln u_{2}} \ln y_{1} \Pi_{|f|}(x) .
\end{aligned}
$$

Если $\ln y_{1} \leqslant \ln u_{2}(\ln z)^{-\gamma}$, то оцениваемая часть суммы равна $O(R)$. Вторая сумма будет

$$
\ll \sum_{\substack{n \leqslant x \\ n_{1}>x^{1 / 3}}}|f(n)| \tau(n-a) \ln x \ll\left(\sum_{n \leqslant x} f^{4}(n)\right)^{1 / 4}\left(\sum_{n \leqslant x} \tau^{4}(n)\right)^{1 / 4}\left(\sum_{x^{1 / 3}<n_{1} \leqslant x} \frac{x}{n_{1}}\right)^{1 / 2} \ln x .
$$

Будем предполагать, что

$$
y_{1}<y_{2} \leqslant \exp \left(\frac{\ln x}{(\ln \ln x)^{2}}\right)
$$

Tak как

$$
\begin{aligned}
\sum_{x^{1 / 3}<n_{1} \leqslant x} \frac{1}{n_{1}} & \ll x^{-1 /\left(3 \ln y_{1}\right)} \sum_{n_{1}} n_{1}^{-1+1 / \ln y_{1}} \ll \exp \left(-\frac{\ln x}{3 \ln y_{1}}+\sum_{p \leqslant y_{1}} p^{-1} \exp \left(\frac{\ln p}{\ln y_{1}}\right)\right) \\
& \ll \exp \left(-\frac{\ln x}{3 \ln y_{1}}+e \ln \ln y_{1}\right),
\end{aligned}
$$

получим при нашем условии на $y_{1}$

$$
\sum_{x^{1 / 3}<n_{1} \leqslant x} \frac{1}{n_{1}} \ll \exp \left(-\frac{1}{4}(\ln \ln x)^{2}\right),
$$

т.е. и эта часть суммы равна $O(R)$. Действуя аналогично, получим, что если $\ln y_{2} \leqslant$ $\ln v_{2}(\ln z)^{-\gamma}$, то часть суммы $\Delta(z)$, в которой $n_{2}>v_{2}$, будет равна $O(R)$. Пусть $v=u_{2} v_{2}$. Если $n_{3} \leqslant x /(v \ln \gamma z)$, то $n_{1} n_{2} n_{3} \leqslant x /\left(\ln ^{\gamma} z\right)$, и вклад таких слагаемых после применения леммы 1 будет равен $O(R)$. Подводя итог, при $z \leqslant \exp \left((\ln \ln x)^{2}\right)$ получим

$$
\Delta(z) \leqslant \sum_{d_{3} \leqslant Q} \frac{1}{\varphi\left(d_{3}\right)} \sum_{x /\left(v \ln ^{\gamma} z\right)<n_{3} \leqslant x /\left(u_{1} v_{1}\right)}\left|f\left(n_{3}\right)\right| \delta\left(z, d_{3}, n_{3}\right)+R
$$

где

$$
\delta\left(z, d_{3}, n_{3}\right)=\sum_{d_{1} d_{2} \leqslant Q / d_{3}}^{\prime}\left|\sum_{n_{3} n_{1} n_{2} \equiv a\left(\bmod d_{1} d_{2}\right)}^{\prime \prime} f\left(n_{1} n_{2}\right)-\frac{1}{\varphi\left(d_{2}\right)} \sum_{\substack{n_{3} n_{1} n_{2} \equiv a\left(\bmod d_{1}\right) \\\left(n_{1} n_{2}, d_{2}\right)=1}}^{\prime \prime} f\left(n_{1} n_{2}\right)\right| .
$$


Здесь $\sum^{\prime}$ означает, что $d_{1} d_{2} \leqslant u,\left(d_{1} d_{2}, a n_{3}\right)=1, \sum^{\prime \prime}$ означает, что $n_{1} n_{2} n_{3} \leqslant x$, $u_{1}<n_{1} \leqslant u_{2}, v_{1}<n_{2}$. Возьмем

$$
\begin{array}{cl}
y_{1}=\exp \left(\ln ^{\gamma_{1}} z\right), \quad y_{2}=\exp \left(\ln ^{\gamma_{2}} z\right), & u_{1}=\exp \left(\ln ^{\sigma_{1}} z\right), \quad u_{2}=\exp \left(\ln ^{\sigma_{2}} z\right), \\
v_{1}=\exp \left(\ln ^{\delta_{1}} z\right), \quad v_{2}=\exp \left(\ln ^{\delta_{2}} z\right) .
\end{array}
$$

Условия на параметры примут следуюший вид:

$$
A \sigma_{1} \leqslant \alpha \gamma_{1}-\gamma, \quad A \delta_{1} \leqslant(A+\alpha) \gamma_{2}-\alpha \gamma_{1}-\gamma, \quad \gamma_{1} \leqslant \sigma_{2}-\gamma, \quad \gamma_{2} \leqslant \delta_{2}-\gamma .
$$

Сделаем суммирования по $n_{2}$ и $n_{1}$ независимыми.

С этой целью разобьем промежуток изменения $n_{1}$, т.е. $\left[u_{1}, u_{2}\right)$ на промежутки вида $\mathscr{J}_{l}=\left[u_{1}\left(1+\ln ^{-D} z\right)^{l}, u_{1}\left(1+\ln ^{-D} z\right)^{l+1}\right)$. Число таких промежутков

$$
L=O\left(\ln ^{D} z \cdot \ln \frac{u_{2}}{u_{1}}\right)=O\left((\ln z)^{D+\sigma_{2}+\sigma_{1}}\right) .
$$

Когда $n_{1} \in \mathscr{J}_{l}$, будем считать, что $n_{2} \leqslant x /\left(n_{3} M_{l}\right)$, где $M_{l}=u_{1}\left(1+\ln ^{-D} z\right)^{l}$. Возникающая при этом ошибка в сумме $\Delta(z)$ будет

$$
\begin{aligned}
& \ll L \max _{l} \sum_{\substack{d_{1} d_{2} d_{3} \leqslant Q \\
\left(d_{1} d_{2}, a\right)=1}} \frac{1}{\varphi\left(d_{3}\right)} \sum_{n_{1} \in \mathscr{J}_{l}}\left|f\left(n_{1}\right)\right|\left(\sum_{\substack{x / M_{l+1}<n_{2} n_{3} \leqslant x / M_{l} \\
n_{3} n_{1} n_{2} \equiv a\left(\bmod d_{1} d_{2}\right)}}\left|f\left(n_{2} n_{3}\right)\right|\right. \\
& \left.+\frac{1}{\varphi\left(d_{2}\right)} \sum_{\substack{x / M_{l+1}<n_{2} n_{3} \leqslant x / M_{l} \\
n_{3} n_{1} n_{2} \equiv a\left(\bmod d_{1}\right)}}\left|f\left(n_{2} n_{3}\right)\right|\right) .
\end{aligned}
$$

После применения леммы 1 получим, что эта ошибка

$$
\begin{aligned}
& \ll \frac{L \Pi_{|f|}(x)}{\ln ^{D} z} \max _{l} \frac{1}{M_{l+1}} \sum_{n_{1} \in \mathscr{J}_{l}}\left|f\left(n_{1}\right)\right| \\
& \leqslant x \ln ^{\sigma_{2}} z \Pi_{|f|}(x) \max _{l}\left(\frac{1}{M_{l+1}} \sum_{n_{1} \leqslant M_{l+1}} f^{2}\left(n_{1}\right)\right)^{1 / 2} \ln ^{-D / 2} z \\
& \ll x(\ln z)^{\sigma_{2}+A^{2} \gamma_{1} / 2-D / 2} \ll R,
\end{aligned}
$$

если $D / 2>\sigma_{2}+A^{2} \gamma_{1} / 2+\gamma$. Таким образом, задача свелась к оценке

$$
\begin{gathered}
\delta_{l}^{\prime}\left(z, d_{3}, n_{3}\right)=\sum_{d_{1} d_{2} \leqslant u}^{\prime} \mid \sum_{\substack{n_{1} \in \mathscr{J}_{l} \\
\left(n_{1}, d_{2}\right)=1}} f\left(n_{1}\right)\left(\sum_{\substack{v_{1}<n_{2} \leqslant x /\left(n_{3} M_{l}\right) \\
n_{1} n_{3} n_{2} \equiv a\left(\bmod d_{1} d_{2}\right)}} f\left(n_{2}\right)\right. \\
\left.-\frac{1}{\varphi\left(d_{2}\right)} \sum_{\substack{v_{1}<n_{2} \leqslant x /\left(n_{3} M_{l}\right) \\
n_{1} n_{3} n_{2} \equiv a\left(\bmod d_{1}\right) \\
\left(n_{2}, d_{2}\right)=1}} f\left(n_{2}\right)\right) \mid \\
\leqslant \sum_{d_{1} d_{2} \leqslant u} \frac{1}{\varphi\left(d_{1} d_{2}\right)} \sum_{\substack{\chi_{d_{1}}, \chi_{d_{2}} \\
\chi_{d_{2}} \neq \chi_{0}}}\left|\sum_{n_{1} \in \mathscr{J}_{l}} \chi_{d_{1} d_{2}\left(n_{1}\right) f\left(n_{1}\right)} \chi_{d_{1} d_{2}}(n) f\left(n_{2}\right)\right| \\
\sum_{v_{1}<n_{2} \leqslant x /\left(n_{3} M_{l}\right)}
\end{gathered}
$$


Перейдем к примитивным характерам. Так как $\chi_{d_{2}} \neq \chi_{0}$ и $\left(d_{2}, P(\sqrt{z})\right)=1$, модуль примитивного характера, порождающего $\chi_{d_{1}} \chi_{d_{2}}$, будет $\geqslant \sqrt{z}$. Следовательно, получим

$$
\delta_{l}^{\prime}\left(z, d_{3}, n_{3}\right) \leqslant \sum_{\sqrt{z}<\delta \leqslant u} \frac{1}{\varphi(\delta)} \sum_{\substack{m \mid P(z) \\ m \leqslant u / \delta}} \frac{1}{\varphi(m)} \sum_{\chi_{\delta}^{*}}\left|S_{l}\left(\chi_{\delta}^{*}\right)\right| \cdot\left|F_{l}\left(\chi_{\delta}^{*}\right)\right|
$$

где

$$
\begin{gathered}
S_{l}\left(\chi_{\delta}^{*}\right)=\sum_{\substack{n_{1} \in \mathscr{F}_{l} \\
\left(n_{1}, m d_{3}\right)=1}} \chi_{\delta}^{*}\left(n_{1}\right) f\left(n_{1}\right), \\
F_{l}\left(\chi_{\delta}^{*}\right)=\sum_{\substack{v_{1}<n_{2} \leqslant x /\left(n_{3} M_{l}\right) \\
\left(n_{2}, m d_{3}\right)=1}} \chi_{\delta}^{*}\left(n_{2}\right) f\left(n_{2}\right) .
\end{gathered}
$$

Разобьем промежуток изменения $\delta$, т.е. $[\sqrt{z}, u)$, на промежутки $N_{t}=\left[2^{t} \sqrt{z}, 2^{t+1} \sqrt{z}\right)$. В результате получим

$$
\begin{aligned}
& \delta_{l}^{\prime}\left(z, d_{3}, n_{3}\right) \ll \ln \frac{u}{\sqrt{z}} \max _{l} \frac{1}{2^{t} \sqrt{z}} \sum_{\delta \in N_{t}} \frac{\delta}{\varphi(\delta)} \sum_{\substack{m \leqslant u / \delta \\
m \mid P(z)}} \frac{1}{\varphi(m)} \sum_{\chi_{\delta}^{*}}\left|S_{l}\left(\chi_{\delta}^{*}\right)\right| \cdot\left|F_{l}\left(\chi_{\delta}^{*}\right)\right| \\
& \ll\left(\ln ^{\gamma+2} z\right) \max _{t, m} \frac{1}{2^{t} \sqrt{z}}\left(\sum_{\delta \in N_{t}} \frac{\delta}{\varphi(\delta)} \sum_{\chi_{\delta}^{*}}\left|S_{l}\left(\chi_{\delta}^{*}\right)\right|^{2}\right)^{1 / 2}\left(\sum_{\delta \in N_{t}} \frac{\delta}{\varphi(\delta)} \sum_{\chi_{\delta}^{*}}\left|F_{l}\left(\chi_{\delta}^{*}\right)\right|^{2}\right)^{1 / 2} .
\end{aligned}
$$

Применяя неравенство большого решета (см. [9, теорема 2.5]), получим

$$
\begin{gathered}
\delta_{l}^{\prime}\left(z, d_{3}, n_{3}\right) \ll \ln ^{\gamma+2} z \cdot \max _{t} \frac{1}{2^{t} \sqrt{z}}\left(\left(\left(2^{t} \sqrt{z}\right)^{2}+\frac{M_{l}}{\ln ^{D} z}\right)\right. \\
\left.\times \sum_{M_{l}<n_{1} \leqslant M_{l+1}}\left|f^{2}\left(n_{1}\right)\right|\left(\left(2^{t} \sqrt{z}\right)^{2}+\frac{x}{M_{l} n_{3}}\right) \sum_{n_{2} \leqslant x /\left(M_{l} n_{3}\right)}\left|f^{2}\left(n_{2}\right)\right|\right)^{1 / 2} \\
\ll \ln { }^{\gamma+2} z \cdot\left(\sum_{n_{1} n_{2} \leqslant 2 x / n_{3}}\left|f^{2}\left(n_{1} n_{2}\right)\right|\right)^{1 / 2}\left(u+\sqrt{u_{2}}+\frac{1}{\sqrt{u_{1}}} \frac{\sqrt{x}}{\sqrt{n_{3}}}+\frac{1}{\sqrt{z}} \frac{\sqrt{x}}{\sqrt{n_{3}}}\right) .
\end{gathered}
$$

Таким образом,

$$
\begin{aligned}
\Delta(z) \ll & (\ln z)^{D+\sigma_{2}+\gamma+2} \sum_{d_{3} \leqslant Q} \frac{1}{\varphi\left(d_{3}\right)} \sum_{x /(v \ln \gamma}\left|f\left(n_{3}\right)\right| \\
& \times\left(\sum_{n_{1} n_{2} \leqslant 2 x / n_{3}}\left|f^{2}\left(n_{1} n_{2}\right)\right|\right)^{1 / 2}\left(u+\sqrt{u_{2}}+\frac{1}{\sqrt{u_{1}}} \frac{\sqrt{x}}{\sqrt{n_{3}}}+\frac{1}{\sqrt{z}} \frac{\sqrt{x}}{\sqrt{n_{3}}}\right) .
\end{aligned}
$$

Так как $n_{3} \leqslant x /\left(u_{1} v_{1}\right)$, то

$$
\frac{1}{\sqrt{u_{1}}} \frac{\sqrt{x}}{\sqrt{n_{3}}} \geqslant \sqrt{v_{1}}>\exp \left(\frac{1}{2} \ln ^{\delta_{1}} z\right)>\sqrt{u_{2}}
$$


при $\delta_{1}>\sigma_{2}$. Кроме того, $\sqrt{x} / \sqrt{u_{1} n_{3}}>u$ при $\delta_{1}>1+\gamma$. Эти предположения не противоречат ранее принятьм условиям на параметры. Поэтому

$$
\Delta(z) \ll(\ln z)^{D+\sigma_{2}+\gamma+1} \frac{x \ln x}{\sqrt{u_{1}}} \sum_{x /(v \ln \gamma} \sum_{z)<n_{3} \leqslant x /\left(v_{1} u_{1}\right)} \frac{\left|f\left(n_{3}\right)\right|}{n_{3}} \exp \left(\frac{1}{2} \sum_{p \leqslant y_{2}} \frac{\left|f^{2}(p)\right|}{p}\right) .
$$

Отсюда, учитывая, что

$$
\begin{aligned}
\sum_{x /\left(v \ln ^{\gamma} z\right)<n_{3} \leqslant x /\left(v_{1} u_{1}\right)} \frac{\left|f\left(n_{3}\right)\right|}{n_{3}} & \leqslant \ln \frac{2 v \ln ^{\gamma} z}{v_{1} u_{1}} \sum_{x /(v \ln \gamma} \max _{z) \leqslant T \leqslant x /\left(v_{1} u_{1}\right)} \frac{1}{T} \sum_{n_{3} \leqslant 2 T}\left|f\left(n_{3}\right)\right| \\
& \ll \ln \frac{2 v \ln ^{\gamma} z}{v_{1} u_{1}} \Pi_{|f|}(x),
\end{aligned}
$$

получаем

$$
\Delta(z) \ll x(\ln z)^{B_{1}} \exp \left(-\frac{1}{2} \ln ^{\sigma_{1}} z\right) \exp \left(A^{2} \ln \ln y_{2}\right) \Pi_{|f|}(x) \ll R
$$

Пусть теперь $\exp \left((\ln \ln x)^{2}\right)<z \leqslant Q$. В этом случае представим $n$ в виде $n=n_{1} n_{2}$, где $n_{1} \mid P\left(y_{1}\right)$, а $\left(n_{2}, P\left(y_{1}\right)\right)=1$. Действуя, как и ранее, мы можем считать, что $u_{1}<$ $n_{1} \leqslant u_{2} \leqslant x^{1 / 3}, n_{2} \geqslant x /\left(u_{2} \ln ^{\gamma} z\right)=v_{1}$, где

$$
\left(\ln u_{1}\right)^{\Delta} \leqslant\left(\ln y_{1}\right)^{\alpha}(\ln z)^{-\gamma}, \quad y_{1}=\exp \left(\frac{\ln x}{(\ln \ln x)^{2}}\right) .
$$

Возьмем $u_{1}=\exp \left((\ln \ln x)^{2}\right)$. Область изменения $n_{1}$ разобьем на промежутки $\mathscr{J}_{l}=$ $\left[u_{1}\left(1+(\ln x)^{-D}\right)^{l}, u_{1}\left(1+(\ln x)^{-D}\right)^{l+1}\right)$. Число промежутков $L=O\left((\ln x)^{D+1}\right)$. Когда $n_{1} \in \mathscr{J}_{l}$, будем считать, что $n_{2} \leqslant x / M_{l}$, где $M_{l}=u_{1}\left(1+(\ln x)^{-D}\right)^{l}$, а не $n_{2} \leqslant x / n_{1}$. Возникающая при этом ошибка будет

$$
\begin{aligned}
& \ll L \max _{l} \sum_{\substack{d_{1} d_{2} d_{3} \leqslant Q \\
\left(d_{1} d_{2} d_{3}, a\right)=1}} \frac{1}{\varphi\left(d_{3}\right)} \sum_{n_{1} \in \mathscr{J}_{l}}\left|f\left(n_{1}\right)\right|\left(\sum_{\substack{x / M_{l+1}<n_{2} \leqslant x / M_{l} \\
n_{1} n_{2} \equiv a\left(\bmod d_{1} d_{2}\right)}}\left|f\left(n_{2}\right)\right|\right. \\
& \left.+\frac{1}{\varphi\left(d_{2}\right)} \sum_{\substack{x / M_{l+1}<n_{2} \leqslant x / M_{l} \\
n_{1} n_{2} \equiv a\left(\bmod d_{1}\right)}}\left|f\left(n_{2}\right)\right|\right) .
\end{aligned}
$$

После применения леммы 1 получим, что эта ошибка

$$
\begin{aligned}
& \ll x \ln x \max _{l} \frac{1}{M_{l}} \sum_{n_{1} \in \mathscr{J}_{l}}\left|f\left(n_{1}\right)\right| \Pi_{|f|}(x) \\
& \ll x \ln x \max _{l}\left(\frac{1}{M_{l+1}} \sum_{n_{1} \leqslant M_{l+1}}\left|f\left(n_{1}\right)\right|^{2}(\ln x)^{-D}\right)^{1 / 2} \\
& \ll x(\ln x)^{1+A^{2} / 2-D / 2} \Pi_{|f|}(x) \ll R,
\end{aligned}
$$


если $D \geqslant A^{2}+1+2 \gamma$.

Далее, как и ранее, используя характеры, избавимся от условия, что $n_{1} n_{2}$ входит в прогрессии. Затем перейдем к примитивным характерам. В результате получим

$$
\Delta(z) \ll L \max _{l} \sum_{m \leqslant x} \frac{1}{\varphi(m)} \sum_{\sqrt{z}<\delta \leqslant Q} \frac{1}{\varphi(\delta)} \sum_{\chi_{\delta}^{*}}\left|S_{l}\left(\chi_{\delta}^{*}\right)\right| \cdot\left|F_{l}\left(\chi_{\delta}^{*}\right)\right|+R,
$$

где

$$
S_{l}\left(\chi_{\delta}^{*}\right)=\sum_{\substack{n_{1} \in \mathscr{J}_{l} \\\left(n_{1}, m\right)=1}} \chi_{\delta}^{*}\left(n_{1}\right) f\left(n_{1}\right), \quad F_{l}\left(\chi_{\delta}^{*}\right)=\sum_{\substack{v_{1}<n_{2} \leqslant x / M_{l} \\\left(n_{2}, m\right)=1}} \chi_{\delta}^{*}\left(n_{2}\right) f\left(n_{2}\right) .
$$

Разбив промежуток изменения $\delta$ на промежутки вида $N_{t}=\left[2^{t} \sqrt{z}, 2^{t+1} \sqrt{z}\right)$, применив неравенство Коши и неравенство большого решета, получим

$$
\begin{aligned}
\Delta(z) \ll & (\ln x)^{D+2} \max _{l} \max _{t} \frac{1}{2^{t} \sqrt{z}}\left(\left(2^{t} \sqrt{z}\right)^{2}+M_{l+1}\right) \\
& \times\left(\sum_{n \leqslant M_{l+1}}|f(n)|^{2}\left(\left(2^{t} \sqrt{z}\right)^{2}+\frac{x}{M_{l}}\right) \sum_{n \leqslant x / M_{l}}|f(n)|^{2}\right)^{1 / 2} \\
\ll & (\ln x)^{D+2+A^{2}}\left(\left(Q+\sqrt{u_{2}}\right) \sqrt{x}+\frac{x}{\sqrt{u_{1}}}+\frac{x}{\sqrt{z}}\right) \ll \frac{x}{\ln ^{\gamma} x},
\end{aligned}
$$

если $B \geqslant D+2+A^{2}+\gamma \geqslant A^{2}+1+2 \gamma+2+A^{2}+\gamma=3+2 A^{2}+3 \gamma$. Лемма 2 доказана.

ЛЕмма 3. Пусть $f(n)$ - комплекснозначная мультипликативная функция, $|f(n)|$ удовлетворяет условиям теоремы, $a$ - фиксированное число. Тогда при $Q \leqslant \sqrt{x} \times$ $(\ln x)^{-B}, B \geqslant 3+2 A^{2}+3 \gamma$, имеем

$$
\begin{aligned}
\widetilde{\delta}(z) & =\sum_{\substack{d_{1} d_{2} \leqslant Q, d_{1} \mid P(z) \\
\left(d_{2}, P(z)\right)=1,\left(d_{1} d_{2}, a\right)=1}}\left|\sum_{\substack{n \leqslant x \\
n \equiv a\left(\bmod d_{1} d_{2}\right)}} f(n)-\frac{1}{\varphi\left(d_{2}\right)} \sum_{\substack{n \leqslant x \\
n \equiv a\left(\bmod d_{1}\right) \\
\left(n, d_{2}\right)=1}} f(n)\right| \\
& \ll \frac{x}{\ln ^{\gamma} z} \Pi_{|f|}(x) .
\end{aligned}
$$

ДокАЗАТЕльство. Представим $d_{2}$ в виде $d_{2}^{\prime} d_{3}$, где $d_{2}^{\prime} \mid P\left(z^{2}\right),\left(d_{2}^{\prime}, P(z)\right)=1$, $\left(d_{3}, P\left(z^{2}\right)\right)=1$. Тогда

$$
\begin{gathered}
\frac{1}{\varphi\left(d_{2}\right)} \sum_{\substack{n \leqslant x \\
n \equiv a\left(\bmod d_{1}\right) \\
\left(n, d_{2}\right)=1}} f(n)=\frac{1}{\varphi\left(d_{3}\right)} \sum_{\substack{n \leqslant x \\
n \equiv a\left(\bmod d_{1} d_{2}^{\prime}\right) \\
\left(n, d_{3}\right)=1}} f(n)-\frac{1}{\varphi\left(d_{3}\right)} \sum_{\substack{n \leqslant x \\
n \equiv a\left(\bmod d_{1} d_{2}^{\prime}\right) \\
\left(n, d_{3}\right)=1}} f(n) \\
+\frac{1}{\varphi\left(d_{3} d_{2}^{\prime}\right)} \sum_{\substack{n \leqslant x \\
n \equiv a\left(\bmod d_{1}\right) \\
\left(n, d_{2}^{\prime} d_{3}\right)=1}} f(n) .
\end{gathered}
$$


Следовательно, $\widetilde{\delta}(z) \leqslant \widetilde{\delta}\left(z^{2}\right)+\Delta(z)$. Повторив это $k$ раз, имеем

$$
\widetilde{\delta}(z) \leqslant \widetilde{\delta}\left(z^{2^{k}}\right)+\Delta(z)+\Delta\left(z^{2}\right)+\cdots+\Delta\left(z^{2^{k-1}}\right) .
$$

Подставляя из леммы 2 оценку

$$
\Delta\left(z^{2^{k}}\right) \ll \frac{x \Pi_{|f|}(x)}{2^{\gamma k} \ln ^{\gamma} z}
$$

и учитьвая, что при $2^{k} \geqslant \ln Q / \ln z \widetilde{\delta}\left(z^{2^{k}}\right)=0$, получим утверждение леммы 3 .

ДоКАЗАТЕЛЬСТво ТЕОРЕМЫ. Представив $\tau(n-1)$ как сумму по делителям и просуммировав под гиперболой, получим

$$
S(f, x)=2 \sum_{d \leqslant Q} \sum_{\substack{n \leqslant x \\
n \equiv 1(\bmod d)}} f(n)+O\left(\sum_{Q<d \leqslant \sqrt{x}} \sum_{\substack{n \leqslant x \\
n \equiv 1(\bmod d)}} f(n)+\sum_{\substack { d \leqslant \sqrt{x} \\
\begin{subarray}{c}{n \leqslant \sqrt{x} d \\
n \equiv 1(\bmod d){ d \leqslant \sqrt { x } \\
\begin{subarray} { c } { n \leqslant \sqrt { x } d \\
n \equiv 1 ( \operatorname { m o d } d ) } }\end{subarray}} f(n)\right) .
$$

Возьмем $Q=\sqrt{x} / \ln ^{B} x$, где $B \geqslant 3+2 A^{2}+3 \gamma$. Согласно лемме 1 остаточньй член

$$
\ll \frac{1}{\ln x} \Pi_{f}(x)\left(x \sum_{Q<d \leqslant \sqrt{x}} \frac{1}{\varphi(d)}+\sqrt{x} \sum_{d \leqslant \sqrt{x}} \frac{d}{\varphi(d)}\right) \ll \frac{x \ln \ln x}{\ln x} \Pi_{f}(x) .
$$

К главному члену применим лемму 3 . Тогда имеем

$$
S(f, x)=2 \sum_{\substack{d_{1} d_{2} \leqslant Q, d_{1} \mid P(z) \\\left(d_{2}, P(z)\right)=1}} \frac{1}{\varphi\left(d_{2}\right)} \sum_{\substack{n \leqslant x \\ n \equiv 1\left(\bmod d_{1}\right) \\\left(n, d_{2}\right)=1}} f(n)+O(R)
$$

где

$$
R=\frac{x}{\ln ^{\gamma} z} \Pi_{f}(x) .
$$

Часть суммы, в которой $d_{1}>e^{z}$, будет

$$
\ll \frac{x \Pi_{f}(x)}{z \ln x} \sum_{d_{1} d_{2} \leqslant Q} \frac{\ln d_{1}}{\varphi\left(d_{1} d_{2}\right)} \ll x \Pi_{f}(x) \frac{\ln z}{z} .
$$

Во внутренней сумме избавимся от условия $\left(n, d_{2}\right)=1$. Допускаемая ошибка при этом будет

$$
\begin{aligned}
& \ll \sum_{z<p \leqslant Q} \frac{f(p)}{\varphi(p)} \sum_{d_{1} d_{2} \leqslant Q} \frac{1}{\varphi\left(d_{2}\right)} \sum_{\substack{n \leqslant x / p \\
p n \equiv 1\left(\bmod d_{1}\right)}} f(n) \\
& +\sum_{\substack{p^{r} \leqslant y \\
r \geqslant 2, p>z}} \frac{f\left(p^{r}\right)}{p^{r}} \sum_{d_{1} d_{2} \leqslant Q} \frac{1}{\varphi\left(d_{2}\right)} \sum_{\substack{n \leqslant x / p^{r} \\
p^{r} n \equiv 1\left(\bmod d_{1}\right)}} f(n)+\sum_{\substack{n \leqslant x \\
\exists p^{r}, r \geqslant 2, p^{r}>y, p^{r} \mid n}} f(n) \tau(n-1) .
\end{aligned}
$$


Применяя лемму 1 , получим, что две первые суммы равны $O\left(\frac{x}{z} \Pi_{f}(x)\right)$. А к третьей сумме применяем неравенство Коши и, пользуясь теоремой п. 10 из [10], находим, что она

$$
\begin{aligned}
& \ll\left(\sum_{n \leqslant x} f^{4}(n) \sum_{n \leqslant x} \tau^{4}(n)\right)^{1 / 4}\left(\sum_{\substack{\exists \leqslant x \\
\exists p^{r}, r \geqslant 2, p^{r}>y, p^{r} \mid n}} 1\right)^{1 / 2} \\
& \ll\left(x^{2}(\ln x)^{A^{4}+2^{4}}\right)^{1 / 4} x^{1 / 2}\left(\sum_{\substack{p^{r}>y \\
r \geqslant 2}} \frac{1}{p^{r}}\right)^{1 / 2} \ll \frac{x}{\sqrt{y}} \ln ^{A^{4} / 4+4} x .
\end{aligned}
$$

Выбирая $y=(\ln x)^{2 \gamma+A^{4} / 2+8}$, убедимся, что эта ошибка равна $O(R)$. Следовательно,

$$
S(f, x)=2 \sum_{d_{1} d_{2} \leqslant Q}^{\prime} \frac{1}{\varphi\left(d_{2}\right)} \sum_{\substack{n \leqslant x \\ n \equiv 1\left(\bmod d_{1}\right)}} f(n)+O(R) .
$$

Здесь штрих означает, что сумма распространяется по $d_{1} \mid P(z), d_{1} \leqslant e^{z},\left(d_{2}, P(z)\right)=1$. Используя характеры, избавимся от условия, что $n$ входит в прогрессии. Характеры, для которых ряд (3) сходится, обозначим через $\chi^{\prime}$, остальные - через $\chi^{\prime \prime}$.

Тогда можно записать

$$
S(f, x)=2\left(S^{\prime}+S^{\prime \prime}\right)+O(R)
$$

где

$$
\begin{aligned}
S^{\prime} & =\sum_{d_{1} d_{2} \leqslant Q}^{\prime} \frac{1}{\varphi\left(d_{1} d_{2}\right)} \sum_{\chi_{d_{1}}^{\prime}} \sum_{n \leqslant x} \chi_{d_{1}}^{\prime}(n) f(n), \\
S^{\prime \prime} & =\sum_{d_{1} d_{2} \leqslant Q} \frac{1}{\varphi\left(d_{1} d_{2}\right)} \sum_{\chi_{d_{1}}^{\prime \prime}} \sum_{n \leqslant x} \chi_{d_{1}}^{\prime \prime}(n) f(n) .
\end{aligned}
$$

Оценим $S^{\prime \prime}$. Если при $t \neq 0$ сходится ряд

$$
\sum_{p} \frac{f(p)\left(1-\operatorname{Re} \chi_{d_{1}}^{\prime \prime}(p) p^{-i t}\right)}{p}
$$

то сходится ряд, в котором $\chi_{d_{1}}^{\prime \prime}(p) p^{-i t}$ заменено на $\left(\chi_{d_{1}}^{\prime \prime}(p) p^{-i t}\right)^{n}$, где $n$ - натуральное. Возьмем $n$ таким, что $\left(\chi_{d_{1}}^{\prime \prime}(p)\right)^{n}=\chi_{0}(p)$. Тогда получим, что ряд

$$
\sum_{p} \frac{f(p)(1-\cos n t \ln p)}{p}
$$

сходится. Расходимость последнего ряда при $t \neq 0$ следует из леммы 4 [11]. Поэтому согласно теореме 1 [11]

$$
\sum_{n \leqslant x} \chi_{d_{1}}^{\prime \prime}(n) f(n)=o\left(\sum_{n \leqslant x} f(n)\right) .
$$


Отметим, что в [11] теорема 1 доказана при предположении $\left|f\left(p^{r}\right)\right| \leqslant C$. Однако, затем эта теорема была доказана (см. [12, теорема 2]) для более широкого класса, в которьй входят функции $\chi(n) f(n)$, где $f(n)$ удовлетворяет условиям теоремы. Таким образом,

$$
S^{\prime \prime}=o\left(e^{z} \ln x \sum_{n \leqslant x} f(n)\right) .
$$

Если для $\chi_{d_{1}}^{\prime}$ ряд (3) сходится, то для примитивного характера $\chi_{\delta}^{*}$, порождающего характер $\chi_{d_{1}}^{\prime}$, этот ряд также сходится. Однако, число примитивных характеров, для которых ряд (3) сходится, ограничено и будет $\leqslant A / \alpha$. Действительно, пусть $\chi_{\delta_{1}}^{*}, \ldots, \chi_{\delta_{k}}^{*}$ - различные примитивные характеры, для которьх сходится ряд (3). Если $d$ делится на $\delta_{1}, \ldots, \delta_{k}$, то по модулю $d$ найдется $k$ различных характеров $\chi_{1}, \ldots, \chi_{k}$, порожденных $\chi_{\delta_{1}}^{*}, \ldots, \chi_{\delta_{k}}^{*}$. Обозначим через $H$ подгруппу $Z_{d}^{*}$, на которой $\chi_{1}, \ldots, \chi_{k} \equiv 1$. Пусть $G-$ группа характеров $Z_{d}^{*}$ и $G_{1}$ - подгруппа $G$, состоящая из всех характеров $\chi(a)=1$ для всех $a \in H$. Тогда (см., например, [13, с. 474-476]) факторгруппа $Z_{d}^{*} / H$ будет изоморфна группе характеров $G_{1}$. Тем самьм, $\left|Z_{d}^{*} / H\right|=\left|G_{1}\right|$. Так как $\left|G_{1}\right| \geqslant k$, то $|H| \leqslant \varphi(d) / k$.

С другой стороны, из сходимости ряда (3) следует, что

$$
\sum_{\substack{p \leqslant x \\ \chi_{i}(p) \neq 1}} \frac{f(p)}{p}=O(1)
$$

поэтому

$$
\sum_{p \leqslant x} \frac{f(p)}{p}=\sum_{a \in H} \sum_{\substack{p \leqslant x \\ p \equiv a(\bmod d)}} \frac{f(p)}{p}+O(1) \leqslant \frac{A}{k} \ln \ln x+O(1) .
$$

Если $k>A / \alpha$, то последнее противоречит (2). Таким образом, если $\chi_{\delta_{1}}^{*}, \ldots, \chi_{\delta_{k}}^{*}-$ все примитивные характеры, удовлетворяющие условию (3), то

$$
S^{\prime}=\sum_{i=1}^{k} \frac{1}{\varphi\left(\delta_{i}\right)} \sum_{n \leqslant x} \chi_{\delta_{i}}^{*}(n) f(n) \sum_{\substack{\delta_{i} d_{1} d_{2} \leqslant Q \\\left(d_{1}, n\right)=1 \\ \delta_{i} d_{1} \leqslant e^{z}}} \frac{\varphi\left(\delta_{i}\right)}{\varphi\left(\delta_{i} d_{1} d_{2}\right)}
$$

Внутренняя сумма равна

$$
\begin{aligned}
& \left(\sum_{\substack{d_{1} \mid P(z) \\
\left(d_{1}, n\right)=1}} \frac{\varphi\left(\delta_{i}\right)}{\varphi\left(\delta_{i} d_{1}\right)}+O\left(\sum_{d_{1}>e^{z} / \delta_{i}} \frac{1}{\varphi\left(d_{1}\right)}\right)\right)\left(\sum_{d_{1} \mid P(z)} \frac{1}{\varphi\left(d_{1}\right)}+O\left(\sum_{\substack{d_{1}>Q \\
d_{1} \mid P(z)}} \frac{1}{\varphi\left(d_{1}\right)}\right)\right)^{-1} \\
& \times \sum_{\substack{d_{1} \mid P(z) \\
d_{1} \leqslant Q}} \frac{1}{\varphi\left(d_{1}\right)}\left(\sum_{\substack{d_{2} \leqslant Q / d_{1} \\
\left(d_{2}, P(z)\right)=1}} \frac{1}{\varphi\left(d_{2}\right)}+O\left(\sum_{Q /\left(\delta_{i} d_{1}\right)<d \leqslant Q / d_{1}} \frac{1}{\varphi(d)}\right)\right) \\
& =\frac{\zeta(2) \zeta(3) \ln x}{2 \zeta(6)} \prod_{p \mid \delta_{i}} \frac{p(p-1)}{p^{2}-p+1} \prod_{\substack{p \mid n \\
p \leqslant z}} \frac{(p-1)^{2}}{p^{2}-p+1}+O\left(\frac{\ln ^{3} z}{z} \ln x\right) .
\end{aligned}
$$


Здесь мы воспользовались соотношением (см., например, [9, с. 140])

$$
\sum_{d \leqslant Q} \frac{1}{\varphi(d)}=\frac{\zeta(2) \zeta(3)}{2 \zeta(6)} \ln x+O(1)
$$

Следовательно,

$$
S^{\prime}=\frac{\zeta(2) \zeta(3)}{\zeta(6)} \ln x \sum_{i=1}^{k} \frac{1}{\varphi\left(\delta_{i}\right)} \prod_{p \mid \delta_{i}} \frac{p(p-1)}{p^{2}-p+1} \sum_{n \leqslant x} f_{i}(n)
$$

где $f_{i}(n)$ - мультипликативная функция такая, что

$$
f_{i}\left(p^{r}\right)= \begin{cases}\chi_{\delta_{i}}^{*}\left(p^{r}\right) f\left(p^{r}\right) \frac{(p-1)^{2}}{p^{2}-p+1} & \text { при } p \leqslant z \\ \chi_{\delta_{i}}^{*}\left(p^{r}\right) f\left(p^{r}\right) & \text { при } p>z .\end{cases}
$$

Ряды

$$
\sum_{p} \frac{1}{p}\left(f(p)-f_{i}(p)\right)
$$

сходятся, поэтому, применяя теорему 2 из [11], получим

$$
\begin{aligned}
S^{\prime}= & \frac{\zeta(2) \zeta(3)}{\zeta(6)} \ln x \sum_{i=1}^{k} \frac{1}{\varphi\left(\delta_{i}\right)} \prod_{p \mid \delta_{i}} \frac{p(p-1)}{p^{2}-p+1}\left(1+\sum_{r=1}^{\infty} \frac{f\left(p^{r}\right)}{p^{r}}\right)^{-1} \\
& \times \prod_{p \nmid \delta_{i}}\left(1+\sum_{r=1}^{\infty} \frac{\chi_{\delta_{i}}^{*}\left(p^{r}\right) f\left(p^{r}\right)}{p^{r}} \frac{(p-1)^{2}}{p^{2}-p+1}\right)\left(1+\sum_{r=1}^{\infty} \frac{f\left(p^{r}\right)}{p^{r}}\right)^{-1} \\
& \times \prod_{p>z}\left(1+\sum_{r=1}^{\infty} \frac{\chi_{\delta_{i}}^{*}\left(p^{r}\right) f\left(p^{r}\right)}{p^{r}}\right)\left(1+\sum_{r=1}^{\infty} \frac{\chi_{\delta_{i}}^{*}\left(p^{r}\right) f\left(p^{r}\right)}{p^{r}} \frac{(p-1)^{2}}{p^{2}-p+1}\right)^{-1} \\
& \times(1+o(1)) \sum_{n \leqslant x} f(n)+O\left(\frac{x \ln ^{3} z}{z} \Pi_{f}(x)\right) .
\end{aligned}
$$

Из сходимости рядов

$$
\sum_{p} \frac{f(p)}{p}\left(1-\chi_{\delta_{i}}^{*}(p)\right)
$$

следует, что произведение по $p>z$ равно $1+O(\varepsilon(z))$, где $\varepsilon(z) \rightarrow 0$ при $z \rightarrow \infty$.

В работах [11], [12] (см., например, [11, с. 42]) доказано, что если $f(n)$ удовлетворяет условиям теоремы, то

$$
\frac{x}{\ln x} \Pi_{f}(x) \ll \sum_{n \leqslant x} f(n) .
$$

Таким образом, имеем

$$
S(f, x)=C(f) \ln x(1+o(1)+O(\varepsilon(z))) \sum_{n \leqslant x} f(n)
$$

при любом фиксированном $z$, где $\varepsilon(z) \rightarrow 0$ при $z \rightarrow \infty$. Отсюда следует утверждение теоремы. 


\section{СПИСОК ЦИТИРОВАННОЙ ЛИТЕРАТУРЫ}

[1] Линник Ю.В. Дисперсионный метод в бинарных аддитивных задачах. Л.: Изд-во ЛГУ, 1961.

[2] Виноградов А. И. О плотностной гипотезе для $L$-рядов Дирихле // Изв. АН СССР. Сер. матем. 1965. Т. 29. № 4. С. 903-934.

[3] Уфимцева Л. И. Обобщение аддитивной проблемы делителей // Матем. заметки. 1970. T. 7. № 4. C. 477-482.

[4] Бредихин Б. М., Уфимцева Л.И. Бинарные аддитивные задачи и мультипликативные функции // Тр. МИАН. 1972. Т. 128. С. 66-75.

[5] Тимофеев Н. М. Аналог теоремы Халоса в случае обобщения аддитивной проблемы делителей // Матем. заметки. 1990. Т. 48. №1. С. 116-127.

[6] Хрипунова М.Б. Суммы мультипликативных функций со сдвинутыми аргументами // Матем. заметки. 1992. Т. 51. № 4. С. 136-138.

[7] Гедири Х. Асимптотика среднего значения функции делителей по сдвинутым гауссовым числам. Автореферат дисс. ... к. ф.-м.н. М., 1996.

[8] Shiu P. A Brun-Titchmarsh theorem for multiplicative functions // J. Reine Angew. Math. 1980. V. 313. P. 161-170.

[9] Монтгомери Г. Мультипликативная теория чисел. М.: Мир, 1974.

[10] Воронин С. М., Карацуба А. А. Дзета-функция Римана. М.: Физматлит, 1994.

[11] Левин Б. В., Тимофеев Н. М. Теорема сравнения для мультипликативных функций // Acta Arith. 1982. V. 42. P. 21-47.

[12] Туляганов С. Т. Средние значения мультипликативных функций // Матем. заметки. 1991. T. 49. № 5. C. $117-127$.

[13] Боревич З. И., Шафаревич И. Р. Теория чисел. М.: Наука, 1972.

Владимирский педагогический государственный университет

Поступило

Институт математики АН Республики Узбекистан

08.01.97 